\title{
Research on Assessment Method of Grid Frequency Fluctuation Characteristics and Optimization of Speed-governor Parameters
}

\author{
Daohong Lin ${ }^{1}$, Qiang $\mathrm{Wu}^{1}$, Xinshu Wan ${ }^{1}$, Yunqian $\mathrm{Li}^{2}$, Lei Shao ${ }^{2}$ and Hongjun $\mathrm{Zhou}^{3}$ \\ ${ }^{1}$ Hainan Electric Power Company Research Institute, Haikou \\ ${ }^{2}$ Southeast University, Nanjing \\ ${ }^{3}$ Beijing Ponovo Power Co., Ltd, Beijing
}

\begin{abstract}
With the enlargement of power grid scale, connections between different power subsystems in interconnected network have been more-closely related and the analysis of power system dynamic behaviors becomes more complex. This paper selects 3 area indexes which comprehensively reflect frequency change through transient process based on transient frequency stability curve and frequency change ratio curve, and there out builds the quantitative assessment method of frequency fluctuation characteristics. Then a generator set speed governor magnification-targeted optimization model, which determines the optimum of frequency fluctuation characteristics as optimal objective with proposed indexes, is built and solved by multivariate optimization algorithm. At last, an optimal example of practical power grid verifies the feasibility and validity of the proposed assessment method and the optimization model.
\end{abstract}

Keywords-dynamic frequency responses; frequency fluctuation characteristics; frequency stability; multivariate optimization algorithm; speed governor

\section{INTRODUCTION}

With the formation of interconnection pattern of power grid in China, the problems of voltage stability, power angle stability and frequency stability caused by faults are becoming more significant [1]. There is a certain possibility that the interconnected power grid divides into several regional isolated island networks due to the tie line fault. Under these circumstances, the different operation characteristics of different isolated networks and the complexity would make the frequency problem more prominent [2]-[4].

Frequency is one of the important indicators of power grid operation, and it reflects the active power supply-demand balance between generation side and load side of power system [5]. Therefore, the domestic and international research on frequency stability control is widespread at present. In [6] and [7], the frequency characteristics of different regional power grids were discussed and the optimal schemes of underfrequency load-shedding and over-frequency generator-tripping strategies were put forward via time-domain simulation of transient stability; reference [8] analyzed the different influences of power system stabilizer (PSS) selection on frequency stability of islanded power grid. In [9], a preventive correction control system was developed for the frequency control of an offshore island power system, which was used to effectively predict the lowest frequency, frequency changing rate and so on, and thus contributing to the arrangement of generator working schedule and the preparation for generatortripping or load-shedding strategy; reference [10] proposed that the relay protection equipment based on frequency change ratio and the inertia simulation method could be adopted to reduce the frequency instability risk of power grid system; aiming to accurately identify the fault events which would result in isolated island network as early as possible and effectively avoid more losses, reference [11] proposed a hybrid islanding detection technique and a priority-based load-shedding scheme and guaranteed frequency stability by releasing weak current load in the island.

In the above references, it can be found that almost all kinds of research work mainly focus on the frequency control strategies of the risks faced by power grid and isolated island network in engineering practice, but the frequency characteristics optimization schemes were all determined by comparing the visual image of the transient frequency stability curves or comparing the minimum or maximum values of frequency. Nevertheless, the optimization depends not only on the optimization of the frequency value, but also on the time. So there exist some difficulties when selecting the optimal scheme through the above method because that the method is a qualitative method but not a quantitative method.

As is known to all, the frequency control of power system is closely related to the adjustment of governing system, and generator governor control is the key to the initial frequency control [12]-[13]. In [14], the influence of system frequency characteristics and governor parameters on frequency stability was analyzed; reference [15] analyzed the low frequency oscillation in the southeastern part of the European power system, which explored the influence of governor parameters, especially the dead zone of speed deviation, on oscillation damping. It is the consensus of the current research to optimize the dynamic frequency characteristics by adjusting governor parameters. Consequently, after establishing a quantitative assessment method for dynamic frequency fluctuation characteristics, the governor parameters could be adjusted to optimize the characteristics according to the method.

This paper is organized as follows. In Section II, three indexes related to the frequency curve are selected and the 
quantitative assessment method of frequency fluctuation characteristics is constructed based on the indexes. In Section III, a generator set speed governor magnification-targeted optimization model, which determines the optimum of frequency fluctuation characteristics as optimal objective, is built. In Section IV, the proposed model is solved by multivariate optimization algorithm (MOA). In Section V, an optimal example of practical power grid verifies the feasibility and validity of the proposed method based on assessment indexes and the optimization model. Section VI concludes the paper and provides recommendations for further work.

\section{ASSESSMENT INDEXES OF FREQUENCY FLUCTUATION CHARACTERISTICS}

Frequency stability can be divided into short-term transient frequency stability and long-term frequency stability according to time scale. The former mainly evaluates whether the frequency of power system in the transient process satisfies the short-term security and stability constraints of system and equipment and whether the frequency will continue to decline and cause frequency collapse. The latter mainly assesses whether the stable frequency of new steady state meets the requirements of long-time operation of power system after transient process [16]. This paper considers the dynamic frequency response under the condition that the frequency of power grid system stays stable after a fault disturbance in a long term, and assesses the dynamic frequency fluctuation characteristics and further selects the evaluation indexes. If the energy generated by generation side is less than the energy consumed at load side of power system when the fault occurs $\left(W_{\text {gen }}<W_{\text {load }}\right)$, there will be a substantial drop in frequency due to power imbalance. Conversely, if the energy generated by generation side is greater than the energy consumed at load side of power system when the fault occurs $\left(W_{\text {gen }}>W_{\text {load }}\right)$, the system frequency will increase considerably. The greater the power difference between supply and demand sides is, the more serious the power imbalance is, and the frequency curve will fall or rise more sharply. Frequency stability problems occur mostly in isolated island networks, and generally speaking, low-frequency problems are more extensive and typical than high-frequency ones, so the following will exclusively consider the situation of frequency drop after failure. In this case, after the initial frequency dip, the frequency curve enters the oscillation regime and its oscillation amplitude will continue to reduce until it finally reaches the new frequency state and stabilizes.

In transient frequency stability curve $f(t)$, this paper defines $f_{0}$ as the original steady-state frequency, $f_{s}$ as the new steady-state frequency, $t_{0}$ as the time node of fault beginning, $t_{1}$ as the time node when frequency fluctuates for the first time and returns to $f_{s}$ in that process, $t_{2}$ as the time node when frequency enters into new steady-state tolerance range and no longer exceeds limit of the frequency tolerance range for the first time.

The area $S_{1}$ which reflects the frequency-dip situation is chosen as the first evaluation index, which is called frequency dropping index. This index can describe the dropping amplitude and duration of transient frequency and it is mainly affected by synchronous generator and load dynamics and the fast control. The formula of $S_{1}$ is:

$$
S_{1}=\int_{t_{0}}^{t_{1}}\left|f-f_{s}\right| d t
$$

The diagram of $S_{1}$ is shown in Figure I.

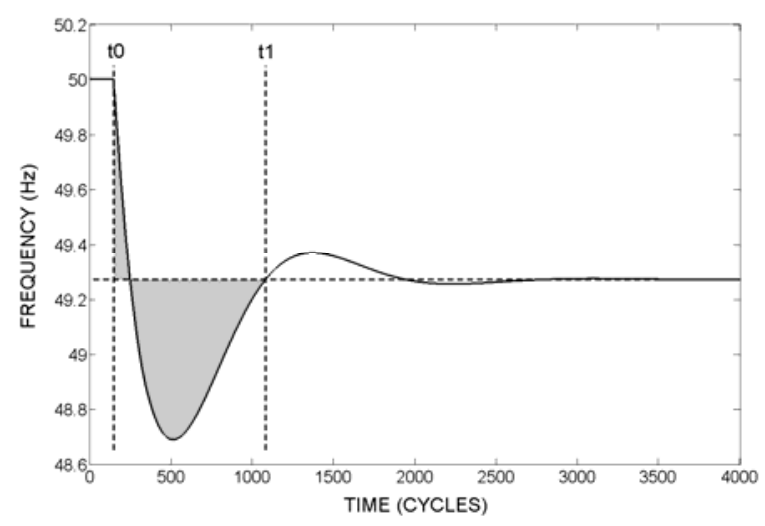

FIGURE I. DIAGRAM OF FREQUENCY DROPPING INDEX

The area $S_{2}$ which reflects the specific situation of frequency oscillation is chosen as the second evaluation index, which is called frequency fluctuation index. The index can describe the amplitude and decay rate of transient frequency oscillation and it is mainly affected by generator governor, load dynamics and the damping intensity of network. The formula of $S_{2}$ is:

$$
S_{2}=\int_{t_{1}}^{t_{2}}\left|f-f_{s}\right| d t
$$

The diagram of $S_{2}$ is shown in Figure II.

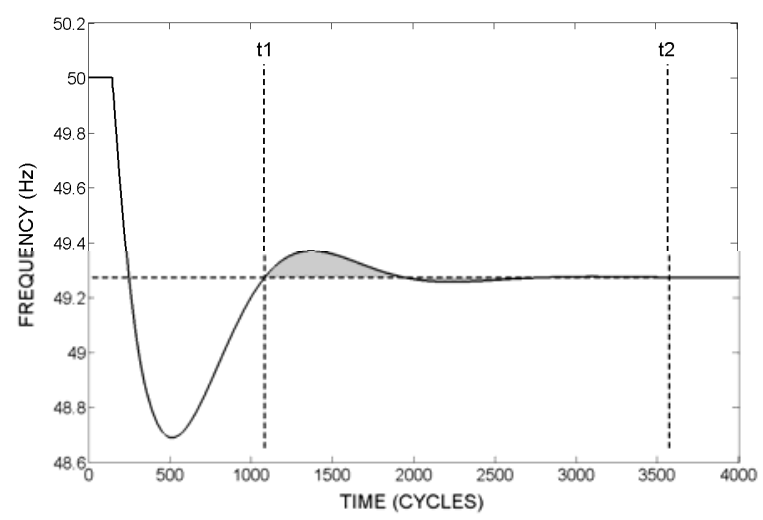

FIGURE II. DIAGRAM OF FREQUENCY FLUCTUATION INDEX 
The area $S_{3}$, which reflects the overall fluctuation situation of transient frequency relative to the steady-state frequency, is considered as the third evaluation index, which is called frequency global moving index. The index is selected from the transient frequency change ratio curve $K_{f}(t)$ and can describe the fluctuation amplitude, the recovery speed after failure and duration of the whole change process of transient frequency, which is mainly affected by the operation mode of power grid before and after the disturbance, the damping intensity of the network and the fast control. The formula of $S_{3}$ is:

$$
\left\{\begin{array}{l}
K_{f}=\frac{d f}{d t} \\
S_{3}=\int_{t_{0}}^{t_{2}}\left|K_{f}\right| d t
\end{array}\right.
$$

The diagram of $S_{3}$ is shown in Figure III.

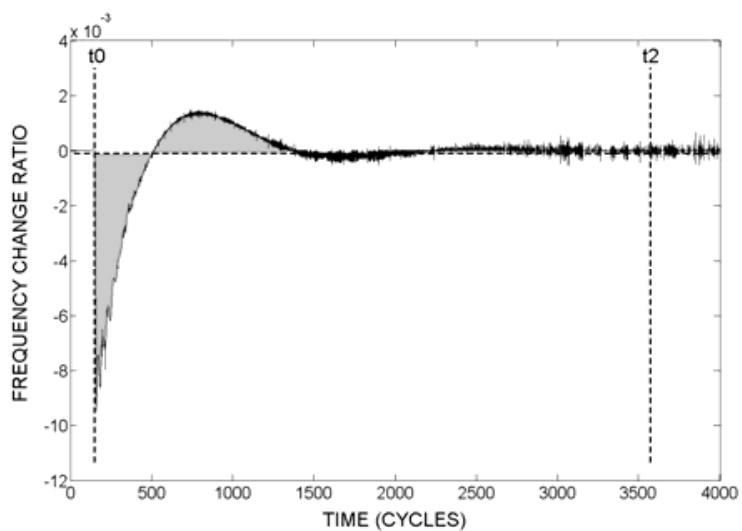

FIGURE III. DIAGRAM OF FREQUENCY GLOBAL MOVING INDEX

The third index is derived from the derivative curve and the derivatives are susceptible to be affected by noises. However, the index is area index and the spikes or glitches of the curve have little effect on index value. Hence, this index can meet the requirement of precision when the requirement of accuracy is not too harsh.

These three indexes are selected from transient frequency stability curve and its derived frequency change ratio curve. By reflecting the two characteristics which are duration and numerical value of frequency or frequency change rate, the whole process of transient frequency change is characterized and described comprehensively. And it is feasible to evaluate the dynamic frequency fluctuation characteristics by using the above indexes as the basic quantitative indicators. In power grid system, when the frequency remains stable, i.e., the frequency change ratio is always in a small range near 0 and there are neither dropping nor fluctuation of frequency, it can be regarded as an ideal state. In this case, the status of indexes above is $S_{1}=S_{2}=S_{3}=0$. According to the above analysis, in the engineering practice, if the assessment indexes of frequency fluctuation characteristics are small, the frequency will be more stable in dynamic process; if the assessment indexes are large, there will be more risks of frequency instability in dynamic process.

\section{Optimization Model of Frequency Fluctuation CHARACTERISTICS}

In this paper, optimization model is established, whose optimization target is the parameter selected from generator governor system and optimization objective is to optimize the dynamic frequency fluctuation characteristics.

\section{A. Governor Parameters Selection}

The governor system of generator sets plays an important role in regulating the frequency of power grid. At present, power system stability analysis mainly uses the standard model provided by IEEE to simulate the governor model, i.e., the model used in BPA which is the main power system simulation software. This paper focuses on the steam turbine governing system whose BPA model is shown in Figure IV.

Since the system is open-loop, the feedback coefficients such as $K_{P}, K_{I}$ and $K_{D}$ will be not considered. Among the remaining adjustable parameters, the speed deviation magnification of governor system is chosen to be optimization target of the model, which is the parameter $K$ in Figure IV.

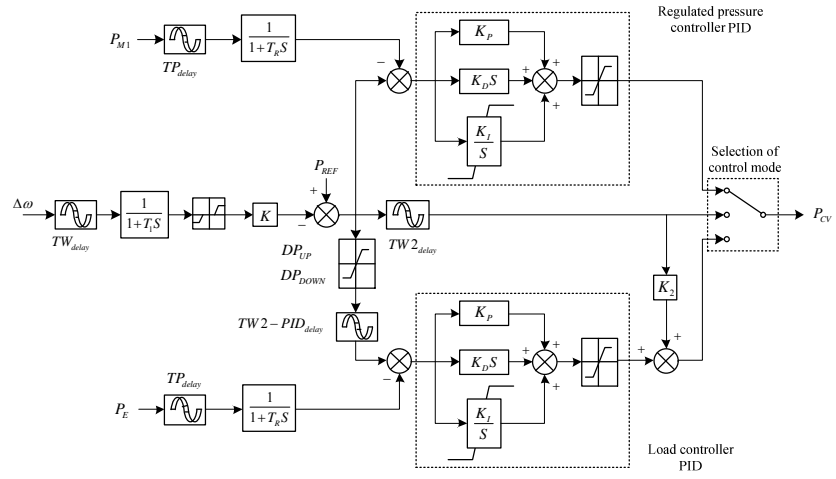

FIGURE IV. THE BPA MODEL OF STEAM TURBINE GOVERNING SYSTEM

\section{B. Objective Function}

According to the above discussion, the frequency curve could be affected by many factors in dynamic process. Without considering the secondary regulating strategy such as underfrequency load-shedding and over-frequency generator-tripping, frequency fluctuation characteristics are still related to the rapid adjustment measures such as governor system of generator sets, load dynamics, the operation mode of grid, damping intensity and so on. The appropriate adjustment of the above factors will have influence on the specific direction of transient frequency stability curve which is further reflected by the indexes. Therefore, in the process of optimizing frequency fluctuation characteristics, four indexes are all set as the optimization objectives. The multi-objective optimization function is constructed as:

$$
F=\left\{\min S_{1}, \min S_{2}, \min S_{3}\right\}
$$


There are various associated factors which could have effects on frequency fluctuation. And the index $S_{1}$ and $S_{2}$ are selected from the same curve and they have the same dimension, so they are transformed to one single objective by linear weighting method. According to the definition of linear weighting method [17], the multi-objective function is transformed into single objective function by:

$$
\left\{\begin{array}{l}
g=\sum_{i=1}^{2} \omega_{i} \times S_{i} \\
\sum_{i=1}^{2} \omega_{i}=1
\end{array}\right.
$$

The target weighting factors are discussed below. Index $S_{1}$ and $S_{2}$ reflect the transient frequency dropping and fluctuation of power grid system. The process of grid frequency fluctuation may bring a lot of harm. It not only threatens the safety of system and generator sets, but also affects power quality of load. Besides, it may also cause malfunction of some relay protection devices [18]. The frequency dropping can be regarded as frequency starting oscillating due to fault, after which the fluctuation amplitude gradually decreases and finally a new steady state will be reached at a certain frequency value. Therefore, frequency dropping and fluctuation can be regarded as homogeneous actions, and it can be defined that $\omega_{1}=\omega_{2}$. According to the equations of weighting factors, the single objective function is calculated and the objective function of the multi-objective optimization model is:

$$
F=\left\{\min g, \min S_{3}\right\}
$$

\section{Constraints}

1) Governor speed deviation magnification constraint

$$
K_{\min } \leq K \leq K_{\max }
$$

In (7), $K$ represents the speed deviation magnification of governor system, while $K_{\min }$ and $K_{\max }$ are respectively the upper and lower limits of the speed deviation magnification of governor system.

\section{2) Speed deviation dead zone constraint}

$$
0 \leq \delta \leq \delta_{\max }
$$

In (8), $\delta$ is the dead zone of speed deviation and $\delta_{\text {max }}$ expresses the predefined maximum critical value of speed dead zone.

\section{3) Generator set power constraint}

$$
\left\{\begin{array}{l}
P_{\min } \leq P_{g e n} \leq P_{\max } \\
Q_{\min } \leq Q_{g e n} \leq Q_{\max }
\end{array}\right.
$$

In (9), $P_{g e n}$ and $Q_{g e n}$ are respectively active and reactive power of the generator set. $P_{\min }$ and $P_{\max }$ are upper and lower limits of active power output of generator. $Q_{\min }$ and $Q_{\max }$ are upper and lower limits of reactive power output of generator.

4) Power balance constraint

$$
P_{\text {gen }, t}=P_{\text {load }, t}
$$

In (10), $P_{\text {gen,t }}$ represents the active power generated by generator set at time $t$ while $P_{\text {load }, t}$ represents the active power consumed by load side at time $t$. The power balance should be guaranteed when power grid operates at any time of original and new steady state.

\section{Multivariate Optimization Algorithm}

Multivariate optimization algorithm (MOA) is a diversified-search intelligent optimization algorithm based on data structure, which divides multiple search elements into global atoms $G_{a}$ and local atoms $L_{a}$. In each iteration, $G_{a}$ is first used to investigate the global situation. Once more potential solutions are found, the information is immediately shared, prompting a new local atom $L_{a}$ to mine the area meticulously. Thus, the solution space is searched efficiently by the alternate global-local search and the global optimum is approached gradually [19]. MOA always maintains the global search field in the process of searching and will not fall into the trap of local convergence and prematurity, which has higher convergence success rate and higher precision compared with other mature population optimization algorithm, so this paper chooses MOA to solve the proposed optimization model.

\section{A. Principles of Multivariate Optimization Algorithm}

An iteration of MOA consists of two stages which are global search and local search. Define the number of global atoms in each iteration as $m$ and the number of local atoms assigned in part $i$ as $m_{i}(1 \leq i \leq m)$. At the beginning of the algorithm, the empty stack is set up to store the global atoms and the potential optimal solutions. In addition, the maximum iteration number is set as $I_{\max }$. In the phase of global searching, global atoms $G_{a}$ are randomly generated in the entire search space which also is the solution space according to the formula that is:

$$
\left\{\begin{array}{l}
G_{a}=\left[h_{1}, \cdots, h_{d}\right] \\
h_{i}=\text { unifrnd }\left(\min _{i}, \max _{i}\right)
\end{array}\right.
$$

In (11), $d$ is the dimension of solution variable; $\min _{i}$ and $\max _{i}$ are the upper and lower bounds of the $i$ th dimension of solution space respectively; unifrnd $\left(\min _{i}, \max _{i}\right)$ indicates that the function returns a random number that is uniform distributed between $\min _{i}$ and $\max _{i}$. Comparing the newly 
generated global atom with global atoms in global stack, the one with better fitness value is chosen as the center of latent solution region to be recorded in global stack of the structure. In the stage of local searching, in order to realize the local search of each potential solution region, local atoms $L_{a}$ are randomly generated in the local neighborhood with the center which is the global atom $G_{a}$ and the radius which is $r$ according to the formula that is:

$$
L_{a}=G_{a}+r \times\left[l_{1}, \cdots, l_{d}\right]
$$

In (12), $l_{i}(1 \leq i \leq d)$ is a random number between -1 and 1 . Comparing the newly generated local atom with local atoms in local stack, the one with better fitness value is selected to retain as historical information.

\section{B. Model Solving Process Based on MOA}

The objective function of the optimization model of network frequency fluctuation characteristics is $F=\left\{\min g, \min S_{3}\right\}$, in which $g$ and $S_{3}$ represent the objective functions derived from transient frequency stability curve and frequency change ratio curve respectively. Since $S_{3}$ can describe the whole change process of dynamic frequency fluctuation relative to the steady state and has unitary meaning, define it as the fitness for comparison in the process of global searching, that is, the indicator of rough searching. Conversely, define $g$ as the fitness for comparison in the process of local searching, i.e., the indicator of meticulous searching.

To sum up, MOA-based solving process of the proposed optimization model of frequency fluctuation characteristics is summarized as follows:

a) Acquire the specific information of the power system lines, load nodes and generator sets, and input the original data of the system.

b) Define an empty global stack and a local stack and define the number of global atoms and local atoms. Define the maximum times of outer iteration and set the current iteration times as 0 . Define the maximum times of inner iteration and set the current iteration times as 0 .

c) Generate each global atom in the search space randomly and then it is plugged into transient stability simulation and calculation program. Determine whether system data meet the model constraints.

d) Select the global atom which has the minimum global fitness value to enter global stack and update global stack. If the fitness value of the new global atom is the minimum in existing global stack, move on to the next step. Otherwise, determine whether the times of inner iteration is up to the upper limit. If the upper limit has been reached, output the current top of local stack as the optimal solution and the program is finished. If not, go back to step c) and the number of inner iteration times is added by 1 .

e) Centering on the newly input global atom, generate each local atom in the local neighborhood with certain radius. Then it is plugged into transient stability simulation and calculation program. Determine whether system data meet the model constraints.

f) Select the local atom which has the minimum local fitness value to enter local stack and update local stack.

g) Determine whether the terminate condition of iterative process has been met, i.e., whether the number of outer iteration times has reached the presupposed maximum number. If so, output the current top of local stack as the optimal solution and the program is finished. If not, the number of outer iteration times is added by 1 and the number of inner iteration times is set as 0 , and then go back to step c) to continue the iteration.

\section{Calculating EXample Analysis}

The actual operation data from Z-S district power system in $\mathrm{J}$ regional grid are used as an example to verify the feasibility and correctness of the proposed assessment method and optimization model. After simulating the dynamic process in which the district grid is disconnected from the main network and come into islanded operation mode due to line-failure by software BPA, it is indicated that the district grid frequency can maintain long-term dynamic stability in winter valley-load operation scene. Therefore, this paper selects this scene to do research.

In the example of Z-S district grid, there are four $220 \mathrm{kV}$ power plants which are named plant 1 to plant 4 in turn. Among them, plant 1, plant 2 and plant 3 each have two generating sets, while plant 4 has four generating sets. The property and parameter configuration of each generator set in the same power plant are approximate, so the research parameters of each generator set in the same power plant are considered the same in this example and will be changed simultaneously in the optimization process. According to engineering experiences, the constraint of governor speed deviation magnification of each generator set is set as $5.0 \leq K \leq 55.0$.

Define the number of global atoms is 5 and the number of local atoms is 10 . The maximum number of outer iteration times is set as 200 while the maximum number of inner iteration times is set as 30 . The dimension of each searching atom is 4 according to the practical example. The optimization model is solved by MOA. The comparison between original and optimal data of governor speed deviation magnification is shown in Table 1 and the comparison between each evaluation index of frequency fluctuation characteristics calculated from original and optimal data is shown in Table 2. According to the original and optimal parameters of speed governor, the transient process is simulated respectively and the transient frequency stability curves are compared in Figure V.

TABLE I. THE ORIGINAL AND OPTIMAL DATA OF GOVERNOR SPEED DEVIATION MAGNIFICATION

\begin{tabular}{|c|c|c|c|c|}
\hline & Plant 1 & Plant 2 & Plant 3 & Plant 4 \\
\hline Origin & 11.7 & 11.7 & 11.7 & 11.7 \\
\hline Optimization & 32.8 & 34.23 & 34.86 & 33.1 \\
\hline
\end{tabular}


TABLE II. THE COMPARISON OF FREQUENCY FLUCTUATION CHARACTERISTICS EVALUATION INDEXES

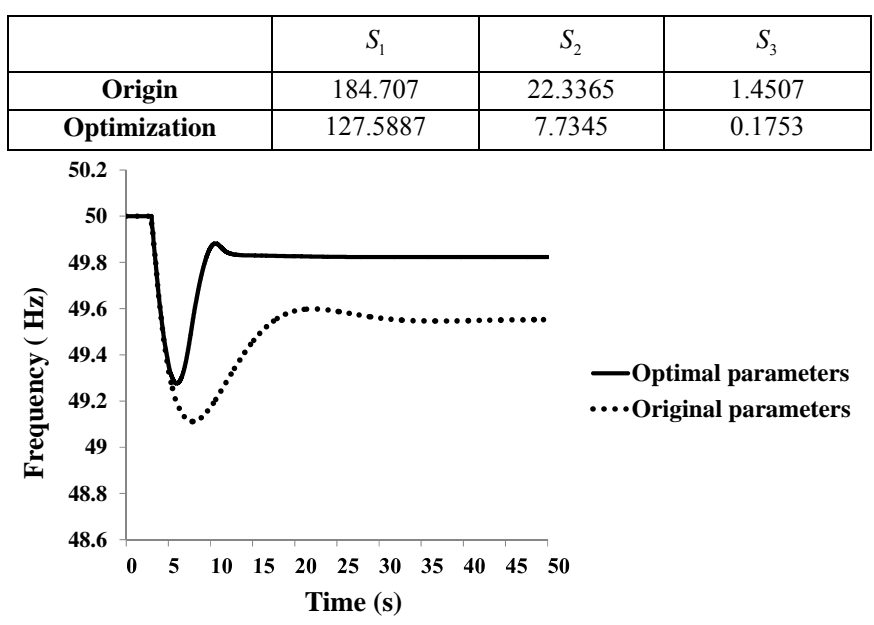

FIGURE V. THE COMPARISON OF TRANSIENT FREQUENCY STABILITY CURVES BASED ON ORIGINAL AND OPTIMAL PARAMETERS

\section{CONCLUSION}

Base on the analysis of dynamic frequency response characteristics which maintains long-term frequency stability after large disturbance caused by fault in power grid, this paper selects 4 area indicators as assessment indexes from transient frequency stability curve and frequency change ratio curve and establishes the quantitative assessment method of frequency fluctuation characteristics, which makes up for the deficiency of intuitive judgment of frequency fluctuation characteristics. Then a generator set speed governor magnification-targeted optimization model, which determines the optimum of frequency fluctuation characteristics as optimal objective, is built and solved by MOA. Finally, in the practical calculating example of Z-S district power system in $\mathrm{J}$ regional grid, the feasibility and validity of the assessment method and the optimization model are verified by using MOA to solve the model. However, there still exists room for improvement and refinement in the field of frequency fluctuation characteristics evaluation method, which needs further study.

\section{ACKNOWLEDGMENT}

The authors gratefully acknowledge the support of the National Natural Science Foundation of China (Study of Regional Interconnected Power Grinds Dynamic Stabilization Corrective Control Based on Network Control, No51277029) and the key project of smart grid technology and equipment of national key research and development plan of china (2016YFB0900602).

\section{REFERENCES}

[1] Hou Yuqiang, Fang Yongjie and Yang Weidong, "A new method of UFLS/UVLS based on multi-agent technology," Automation of Electric Power Systems, vol. 35, no. 4, pp. 1-5, 2011.

[2] Chang Haijun, Huo Chao and Li Wei, "Research on emergency control scheme of isolated power grid based on splitting information triggering," Power System Protection and Control, vol. 44, no. 2, pp. 27-35, 2016.
[3] Zhang Peigao, Li Xingyuan and Li Zheng, "Research on frequency stability and control strategy in isolated power grid," Power System Protection and Control, vol. 40, no. 15, pp. 143-149, 2012.

[4] J. Horne, D. Flynn and T. Littler, "Frequency stability issues for islanded power systems," IEEE PES Power Systems Conference and Exposition, vol. 1, pp. 299-306, 2004.

[5] Zhang Zhen'an, Guo Jinpeng and Zhang Xuemin, "Blackout model and its application considering frequency stability," Proceedings of the CSUEPSA, vol. 27, no. 4, pp. 26-32, 2015.

[6] Zhao Qiang, Wang Limin and Liu Zhaoxu, "Study on dynamic frequency characteristics and coordinative under-frequency load shedding scheme for nationwide interconnected power grid of China," Power System Technology, vol. 33, no. 8, pp. 35-40(in Chinese), 2009.

[7] Hu Jianchen, Hu Feixiong and Li Xian, "Frequency stability and control measures of Hainan power grid after interconnection with China Southern Power Grid," Southern Power System Technology, vol. 3, no. 5 , pp. 31-35, 2009.

[8] Zheng Chao, Chen Xiang, "Influence of Power System Stabilizer on Frequency Stability of Isolated Power Network," Power System Technology, vol. 1, pp. 214-219, 2016.

[9] Y. K. Wu, "Frequency Stability for an Island Power System: Developing an Intelligent Preventive-Corrective Control Mechanism for an Offshore Location," in IEEE Industry Applications Magazine, vol. 23, no. 2, pp. 74-87, March-April 2017.

[10] J. O'Sullivan, A. Rogers, D. Flynn, P. Smith, A. Mullane and M. O'Malley, "Studying the Maximum Instantaneous Non-Synchronous Generation in an Island System-Frequency Stability Challenges in Ireland," in IEEE Transactions on Power Systems, vol. 29, no. 6, pp. 2943-2951, Nov. 2014.

[11] N. K., S. A. Siddiqui and M. Fozdar, "Hybrid islanding detection method and priority-based load shedding for distribution networks in the presence of DG units," in IET Generation, Transmission \& Distribution, vol. 11, no. 3, pp. 586-595, 2017.

[12] Zhu Jizhong, Xu Guoyu, "Study of governor dead-band and generator regulating speed limit in the decentralized load frequency control," Proceedings of the CSEE, vol. 11, no. 1, pp. 15-22, 1991.

[13] Zhu Wei, Tang Ying-jie and Tan Xiyi, "Effect of additional control on frequency stabilization of generator governing system," Electric Power Automation Equipment, vol. 28, no. 12, pp. 21-24, 2008.

[14] He Jingbo, Zhang Jianyun and Li Mingjie, "Frequency domain analysis and control for governor stability problem in islanded HVDC sending systems," Proceedings of the CSEE, vol. 33, no. 16, pp. 137-143(in Chinese), 2013.

[15] J. C. Mantzaris, A. Metsiou and C. D. Vournas, "Analysis of Interarea Oscillations Including Governor Effects and Stabilizer Design in SouthEastern Europe," in IEEE Transactions on Power Systems, vol. 28, no. 4, pp. 4948-4956, Nov. 2013.

[16] Li Changgang, "Studies on assessment and control of power system transient frequency stability," Ph.D. dissertation, Dept. Electrical Engineering, Shandong University, 2012.

[17] Wu Hongbin, Hou Xiaofan and Zhao Bo, "Economical dispatch of microgrid considering plug-in electric vehicles," Automation of Electric Power Systems, vol. 38, no. 9, pp. 77-85, 2014.

[18] He Benteng, Jin Huafeng, "Real-time estimation of power system oscillation period," Automation of Electric Power Systems, vol. 24, no. 15, pp. 32-35(in Chinese), 2000.

[19] Li Baolei, Shi Xinling and Gou Changxing, "Multivariant optimization algorithm and its convergence analysis," Acta Automatica Sinica, vol. 41, no. 5, pp. 949-959, 2015. 